\title{
Evolución de la mortalidad por atropellos y colisiones de tránsito en Argentina entre 2001 y 2010
}

\begin{abstract}
Gabriel Escanés*
En este artículo se analiza la mortalidad vial con el objetivo de medir el impacto que tuvieron los decesos por atropellos y colisiones de tránsito sobre la esperanza de vida de los argentinos entre los trienios 2000-2002 y 2009-2011. Se aplicó el índice de años de esperanza de vida perdidos (AEVP), que sirvió para establecer la cantidad de años que deberían haber vivido las personas que fallecieron por lesiones causadas al transitar por la vía pública, ya sea como peatones, ciclistas, motociclistas u ocupantes de vehículos a motor. Los registros sobre la población segmentada por edad y sexo se tomaron de los dos últimos censos nacionales de población, correspondientes a los años 2001 y 2010. La información sobre las causas de muerte, sexo y edad de los fallecidos se obtuvo de las bases de datos de la Dirección de Estadísticas e Investigación en Salud. Los principales resultados indican que en el trienio 2000-2002 la población argentina perdió 0,59 años (siete meses) de esperanza de vida entre el nacimiento y los 80 años como consecuencia de las muertes en el espacio vial, y 0,66 años (ocho meses) en el trienio 2009-2011.
\end{abstract}

Palabras clave: Colisiones. Tránsito. Atropellos. Esperanza de vida. Mortalidad.

\footnotetext{
* Centro de Investigaciones y Estudios sobre Cultura y Sociedad - Conicet-UNC, Córdoba, Argentina (gaescanes@gmail.com).
} 


\section{Introducción}

Las muertes ocurridas como consecuencia de atropellos a peatones y colisiones de vehículos de transporte terrestre representan un problema de alcance mundial. La Organización Mundial de la Salud (OMS) publicó en 2004 un informe en el que calificaba a este flagelo como una epidemia silenciosa que afectaba a todos los sectores de la sociedad. Según esta publicación, hace una década morían aproximadamente 3.500 personas por día en todo el mundo como consecuencia de lesiones resultantes del tránsito. El $90 \%$ de estos fallecimientos ocurría en los países de ingresos bajos y medios, que concentraban el $48 \%$ del parque vehicular mundial (OMS, 2004).

En 2009, la muerte de 1,27 millones de personas ubicó a los decesos por atropellos y colisiones de tránsito como la octava causa de muerte con mayor impacto en la esperanza de vida de las personas. Si no se toman medidas para frenar su crecimiento exponencial, la cifra de fallecimientos viales alcanzaría los 2,4 millones de personas en 2030, pasando a constituir la quinta causa de muerte más importante a nivel mundial (OMS, 2009). Otras previsiones indican que, en un contexto de ausencia de políticas de seguridad vial, la mortalidad en el tránsito podría aumentar en el mundo en un 65\% para 2020 (PEDEN; TOROYAN, 2004).

Si bien la cantidad de decesos es elevada, la situación se torna aún más compleja al considerar que los traumatismos no mortales alcanzan una cifra anual que se aproxima a los 50 millones de personas heridas como consecuencia de atropellos y colisiones viales. Una encuesta realizada por la OMS reveló que la relación entre muertos y personas con lesiones graves (no mortales) alcanza una razón de 1 sobre 20 . Si se aplicara esta proporción a los 1,27 millones de decesos viales anuales en todo el mundo, se podría estimar en 25,4 millones los heridos graves, es decir, que requieren atención médica en salas de urgencias. La otra mitad de las lesiones que ocurren anualmente a nivel mundial $(24,6$ millones) se consideran leves, ya que no necesitan esa atención de urgencia (OMS, 2009).

Las pérdidas anuales ocasionadas tanto por lesiones como por muertes se estiman en 518.000 millones de dólares americanos, lo que significa que cada país debe destinar entre el $1 \%$ y el $3 \%$ del Producto Bruto Interno a su atención (OMS, 2009). Este elevado costo económico tiene implicaciones negativas para los sistemas de salud, el sector productivo, las víctimas y la sociedad en general.

El mayor riesgo de morir como consecuencia de lesiones causadas por el tránsito corresponde a la región de África, mientras que el menor se registró en el continente europeo, con 24,1 y 10,3 muertes cada 100.000 habitantes, respectivamente. Por su parte, en la región de las Américas la tasa de mortalidad vial asciende a 16,1 muertos cada 100.000 habitantes, aunque cabe mencionar que existen fuertes contrastes entre los países que la componen, sobre todo en el sur. Así, por ejemplo, en Chile y Argentina ocurren 12,3 y 12,6 decesos cada 100.000 habitantes, respectivamente, mientras que en Venezuela el riesgo de muerte vial asciende a 37,2 fallecimientos cada 100.000 habitantes (OMS, 2013). 
En este contexto mundial, caracterizado por una creciente cantidad de muertes ocurridas durante la circulación de las personas, este artículo realiza un análisis demográfico de la evolución de la mortalidad vial en Argentina entre los trienios 2000-2002 y 2009-2011, a fin de poner en evidencia similitudes o diferencias respecto de la situación global. En este sentido, se plantean tres objetivos: a) analizar el nivel y el cambio de la mortalidad vial; b) medir los años de esperanza de vida perdidos (AEVP) como consecuencia de la mortalidad vial, y c) analizar el impacto de los posibles cambios de las tasas de mortalidad vial sobre la esperanza de vida.

Se utiliza una metodología cuantitativa, pues se abordan los datos de manera descriptiva, lo que permite determinar magnitudes y relaciones de la mortalidad vial de acuerdo al sexo de las personas fallecidas, su edad y el país de residencia al momento de su deceso. Los registros de información respecto de las causas de muerte, el sexo y la edad de los fallecidos fueron provistos por los registros civiles provinciales, mientras que los datos sobre la cantidad de personas residentes en el territorio argentino según los censos de 2001 y 2010 se obtuvieron del Instituto Nacional de Estadística y Censos (INDEC).

\section{Antecedentes de la investigación sobre la situación vial en Argentina}

La investigación sobre la situación vial en el país se caracteriza por una prolífica producción de múltiples autores. En este sentido, se pueden encontrar los aportes de corte cuantitativo de trabajos como el de Rey (1999), Foschiatti et al. (2003), Peltzer (2003), Beltramino y Carrera (2007), Prado y Muñoz de la Rosa (2009), Fleitas (2010) y Garcette (2010), entre otros.

En esta misma perspectiva, es posible destacar también las contribuciones de investigaciones realizadas por diferentes instituciones como la Organización Panamericana de la Salud (2007), el Instituto de Seguridad y Educación Vial (2012), la Agencia Nacional de Seguridad Vial (2010) y la Organización Mundial de la Salud (2004, 2009 y 2013), entre otras. Por otra parte, existen algunos trabajos que utilizaron una metodología cualitativa, como el informe publicado por el Defensor del Pueblo de la Nación Argentina (2005), Toledo (2007) y Merlino (2010).

Sobre las medidas de la mortalidad vial en particular, la Comisión Nacional de Programas de Investigación Sanitaria (2006) elaboró un informe referido a los aspectos sociodemográficos de los atropellos y colisiones de tránsito ocurridos entre 1999 y 2002 en Argentina. La publicación indica que en ese período se registró un promedio anual de 4.000 personas fallecidas. La mayoría de las muertes ocurrieron entre los hombres, que triplicaron los fallecimientos femeninos. Además, la mayor frecuencia de decesos se concentró en los jóvenes de entre 15 y 29 años de edad (30\% del total), con un valor máximo en el grupo quinquenal de 20 a 24 años (12\% del total).

El informe indica que la tasa anual de mortalidad vial alcanzó los 11,9 decesos cada 100.000 habitantes. Sin embargo, se observaron grandes diferencias al considerar el sexo 
y la edad de los fallecidos. Los menores de 15 años presentaron las tasas más bajas, y similares entre ambos sexos. Entre los mayores de 15 años, la tasa de mortalidad de los varones creció hasta alcanzar un máximo entre los 20 y los 30 años de edad, con niveles de más de 25 cada 100.000, un crecimiento muy superior al que registraron las mujeres de edades semejantes. Por el contrario, ellas alcanzaron un máximo nivel en sus tasas de mortalidad entre los 15 y los 19 años, el que además se ubicó significativamente por debajo del nivel observado en los hombres de estas edades (COMISIÓN NACIONAL DE PROGRAMAS DE INVESTIGACIÓN SANITARIA, 2006).

Entre 1999 y 2002 los argentinos perdieron casi 170.000 años de vida potencial por colisiones viales, de los cuales 130.000 correspondieron a los hombres y los 40.000 restantes a las mujeres (COMISIÓN NACIONAL DE PROGRAMAS DE INVESTIGACIÓN SANITARIA, 2006).

El fuerte impacto que tiene la mortalidad por atropellos y colisiones de tránsito en la salud y la expectativa de vida de las personas impone la necesidad de diseñar campañas de prevención para reducir las lesiones, las hospitalizaciones, la gravedad de los traumatismos y las muertes (OMS, 2009). En este sentido, los eventos viales que producen consecuencias negativas para la salud de las personas representan un problema de salud pública (MACÍAS; ALMEIDA FILHO; MARCIO, 2010). El próximo apartado hace referencia a las políticas más recientes adoptadas en esta línea a nivel mundial, y particularmente en Argentina.

\section{Los atropellos y colisiones viales: una perspectiva de salud pública}

En 2011 la OMS comenzó a ejecutar el "Plan de Acción del Decenio para la Seguridad Vial 2011-2020”. Esta iniciativa insta a los países de todo el mundo a difundir sus políticas públicas relativas a la seguridad vial (INTERNATIONAL TRAFFIC SAFETY, 2012). La propuesta se apoya en cinco pilares:

- Mejorar la gestión de la seguridad vial: apunta a fortalecer la capacidad institucional para impulsar políticas de alcance nacional.

- Promover mayor seguridad en el tránsito y las rutas: propone mejorar la planificación, el diseño, la construcción y el funcionamiento de calles y rutas, entre otros aspectos.

- Lograr mayor seguridad en los vehículos: promueve la implementación de normas y mecanismos tendientes a la introducción de nuevas tecnologías en el parque automotor.

- Aumentar la eficacia de la respuesta sanitaria luego de los atropellos y colisiones viales: promueve mejoras de la capacidad de los sistemas sanitarios para la atención de las víctimas viales.

- Aumentar la seguridad para los usuarios de la vía pública: aplicar programas de mejora de la conducta de los sujetos en tanto transeúntes. 
A pesar de los esfuerzos de la OMS por definir estos lineamientos útiles para implementar políticas públicas a nivel regional y nacional, la situación de América, y particularmente de América Latina y el Caribe, resulta preocupante por varias razones. Durante el período 2000-2010 algunos países registraron elevados niveles de mortalidad vial, aumentos en el número de fallecidos en el tránsito y reducción de las medidas de control sobre la seguridad vial. En este contexto, se pueden mencionar al menos dos elementos que dificultan la ejecución de políticas públicas vinculadas con esta materia: a) ausencia de leyes integrales, y b) carencia de una institucionalidad específica, por la falta de creación de agencias nacionales (NAZIF; PÉREZ SALAS, 2013).

Si bien todos los países de la región de las Américas cuentan con normas que regulan los límites de velocidad, el consumo de alcohol para conducir, el uso de dispositivos de seguridad (casco y cinturón) y de sillas de retención infantil, ninguno posee leyes integrales en este ámbito (OMS, 2009). Además, si bien hubo un mayor compromiso de los países de la región con la creación de una agencia encargada de la seguridad vial entre 2008 y 2012 -el 89\% de ellos adoptaron esta medida en ese período-, debe advertirse que una mayor institucionalidad vinculada a este tema no necesariamente reduce el riesgo de muerte por sí misma. A modo de ejemplo se puede citar el caso de Colombia, donde se creó la agencia especializada en seguridad vial en 2013, pero antes de eso ya se había logrado reducir la mortalidad vial, de 18,3 a 15,6 muertes cada 100.000 habitantes entre 2000 y 2010 (NAZIF; PÉREZ SALAS, 2013).

En 2008 ya se había registrado en Argentina una iniciativa tendiente a reducir el impacto de las lesiones y muertes ocurridas en atropellos y colisiones viales. Ese año se creó la Agencia Nacional de Seguridad Vial (ANSV), con la finalidad de establecer políticas de promoción, coordinación, control y seguimiento de programas de seguridad vial. Esta institución se hizo responsable de la coordinación y el seguimiento del Plan Nacional de Seguridad Vial (PNSV) (AGENCIA NACIONAL DE SEGURIDAD VIAL, s/d).

EI PNSV estableció cuatro ejes fundamentales para afrontar la problemática vial: concientización, prevención, control y sanción. En este sentido, se han instrumentado políticas públicas orientadas a una multiplicidad de ámbitos, tales como:

- Difusión de campañas en medios de comunicación masiva: se intenta ayudar a la población a reconocer situaciones que potencialmente pueden incrementar los riesgos al transitar de manera imprudente por la vía pública.

- Desarrollo de programas de educación vial: consiste en la entrega de materiales didácticos para el nivel educativo primario y secundario, con el fin de contribuir a la formación ética y ciudadana de niños y jóvenes en los diferentes roles que cumplen en la vía pública.

- Capacitación de autoridades de control y conductores profesionales: se procura reforzar la formación del personal policial y de contralor.

- Control y fiscalización del tránsito. 
- Desarrollo de un sistema de antecedentes de tránsito y estadística accidentológica: se intenta mejorar la calidad de la información recabada en los atropellos y colisiones viales, a partir de la unificación de las estadísticas de seguridad vial.

- Creación de un mapa de riesgo y puntos negros: la finalidad de esta acción se relaciona con la necesidad de prevenir a los usuarios de la vía pública sobre lugares donde se produjeron muertes viales.

- Revisión técnica obligatoria para los vehículos: se intenta asegurar el adecuado funcionamiento del parque automotor.

- Control de la emisión de la licencia nacional de conducir: se propone unificar los criterios de evaluación de aptitudes, conocimientos y capacitación para emitir la licencia.

En este contexto, se plantea en este artículo la necesidad de arrojar luz sobre el estado y la evolución de la mortalidad en ocasión del tránsito de las personas para los trienios 2000-2002 y 2009-2011 en el país. El próximo apartado se ocupa de los lineamientos metodológicos que se siguieron en el análisis de la mortalidad vial.

\section{Metodología de la investigación}

En función del cumplimiento de los objetivos de esta investigación se recurrió al método cuantitativo, con el propósito de medir la mortalidad por atropellos y colisiones de vehículos en Argentina. Esta estrategia consistió en determinar magnitudes y relaciones de la mortalidad vial de acuerdo al sexo de las personas fallecidas, su edad y país de residencia. Se efectuó un estudio transversal en dos momentos: 2000-2002 y 2009-2011, a fin de analizar los cambios ocurridos en la mortalidad entre ambos períodos.

Se consideró como población de estudio a las personas residentes en Argentina entre los trienios 2000-2002 y 2009-2011 que fallecieron como consecuencia de lesiones producidas en atropellos y colisiones de tránsito. Se calculó el promedio trianual de las muertes viales, a fin de suavizar el efecto de posibles fluctuaciones.

Las medidas aplicadas para estudiar el nivel y el cambio de la mortalidad se calcularon sobre la base de dos tipos de información básica: a) el número absoluto de hechos demográficos (defunciones) ocurridos en un período de tiempo, y b) el tamaño de la población relacionada con estos hechos (CENTRO LATINOAMERICANO Y CARIBEÑO DE DEMOGRAFÍA, 1997). La combinación de esta información posibilita la determinación de indicadores (proporciones, tasas, relaciones y probabilidades) que expresan la relación entre variables cuantitativas. Las relaciones más pertinentes en el estudio de la mortalidad por atropellos y colisiones viales se pueden clasificar en indicadores de accidentalidad vinculados a la gestión, el diagnóstico, la evaluación y el control y seguimiento de la situación vial (AGENCIA NACIONAL DE SEGURIDAD VIAL, 2010).

En este artículo se utilizan los indicadores de diagnóstico - las tasas de mortalidad por causa, las tasas de mortalidad específicas por edades y los años de esperanza de vida 
perdidos-, que sirven para dar señales de lo que está sucediendo en el país en relación a la mortalidad vial.

En primer lugar, se consideró la relación entre las víctimas mortales por atropellos y colisiones viales y la población residente en el país, que expresa el riesgo de morir en ocasión de la circulación (como peatón o usuario de los medios de transporte terrestre). En segundo lugar, se contemplaron las tasas de mortalidad específicas por edad vinculadas a los decesos por atropellos y colisiones viales, que sirvieron para medir el nivel de la mortalidad por esta causa en cada grupo etario. Este indicador expresa la frecuencia relativa de muertes, ponderada por una constante de 100.000 habitantes, dentro de grupos de edades de una población en un período de tiempo determinado.

En función de la necesidad de evaluar los diferenciales de mortalidad por sexo, se consideró además la relación de muertes entre hombres y mujeres a partir de las tasas de mortalidad específicas por edad. Esta relación, comúnmente llamada sobremortalidad masculina, se obtuvo mediante el cociente entre las tasas masculinas y las femeninas. De este modo, se midió la cantidad de decesos de hombres por cada fallecimiento femenino ocurridos en Argentina entre los trienios 2000-2002 y 2009-2011.

La utilización de las tasas de mortalidad específicas por edad permitió medir adecuadamente el nivel y el cambio de la mortalidad en cada una de las edades. Sin embargo, su análisis se limitó por dos aspectos: a) las tasas no reflejan el impacto que producen sus cambios sobre la vida promedio de la población, y b) se dificulta la comparación de una población en dos períodos de tiempo diferentes, ya que si en esa comparación las tasas expresan niveles más altos o más bajos en distintas edades, es muy difícil determinar en qué período es mayor la mortalidad general (ARRIAGA, 1996). Además, cabe aclarar que se optó por no estandarizar las tasas de mortalidad en la comparación entre los trienios considerados ${ }^{1}$.

En tercer lugar, dado que los indicadores mencionados no logran medir el nivel y el cambio absoluto y relativo de la mortalidad, se aplicó el índice de años de esperanza de vida perdidos, que sirve para "determinar cuántos años más deberían haber vivido las personas que fallecen" (ARRIAGA, 1996, p. 11). Este índice fue calculado en diferentes países para evaluar el impacto de los atropellos y colisiones en la esperanza de vida de la población (CHANDRAN et al., 2013; LAPOSTOLLE et al., 2009; POLINDER et al., 2007). Dado que no se conoce la cantidad de años que cada persona debería vivir, el concepto de años de esperanza de vida perdidos necesita de algunos supuestos, tales como:

\footnotetext{
${ }_{1}^{1}$ Si bien este procedimiento igualaría los efectos de la estructura etaria en la comparación de la población para ambos períodos, la interpretación de las tasas estandarizadas no es la misma que la de las tasas brutas de mortalidad. Al respecto, Arriaga (2011) explica que los resultados de calcular el riesgo de muerte a partir de una población estándar -para ambos períodos- muestran el rango de las tasas estandarizadas. Por ejemplo, si la tasa de mortalidad estandarizada por atropellos y colisiones viales del trienio 2009-2011 fuera un 14\% mayor que la misma tasa para el trienio 2000-2002, no significaría que la mortalidad fue un 14\% más alta en 2009-2011 que en el primer trienio. Esto se debe a que los resultados dependerán de la población estándar que se utilice en los cálculos. Si se emplean distintas combinaciones de poblaciones consideradas "estándar", los rangos de las tasas estandarizadas se modificarían, razón por la que se aplicará la metodología de años de esperanza de vida perdidos para medir el cambio de la mortalidad.
} 
- "Que la mortalidad debería ser nula entre dos edades elegidas para el análisis. En otros términos, aquellos que mueren, deberían haber vivido hasta la edad superior del intervalo de edades donde se analiza la mortalidad” (ARRIAGA, 1996, p. 11).

- "Que entre las dos edades elegidas para el análisis, aquellas personas que mueren a una edad determinada, de no haber muerto, deberían haber vivido tantos años como el promedio que vive la población que no muere a dicha edad” (ARRIAGA, 1996, p. 11).

- "No limitar la edad superior del análisis, y suponer que aquellos que fallecen a una edad determinada, si no hubieran muerto, habrían vivido tantos años como el resto de la población que queda viva a esa misma edad" (ARRIAGA, 1996, p. 11).

El uso de los años de esperanza de vida perdidos permitió determinar el impacto que tuvieron las muertes por atropellos y colisiones viales sobre la esperanza de vida en el país. Para calcular este indicador fue necesaria la elaboración de tablas de mortalidad, a partir de la cantidad de muertos por grupo de edad y sexo para cada trienio y la cantidad de personas censadas en 2001 y 2010. Estas tablas están disponibles en el anexo de este artículo.

A manera de ejemplo, si la población residente en Argentina en 2010 vivió, entre su nacimiento y los 80 años de edad, un promedio de 69 años (esperanza de vida temporaria), entonces esa población perdió, entre su nacimiento y esa edad, 11 años de esperanza de vida. Estos años de vida que los argentinos no vivieron en 2010 expresan los años de esperanza de vida que el conjunto de muertes hicieron perder entre el nacimiento y los 80 años de edad. En consecuencia, cuanto mayor sea el nivel de la mortalidad, particularmente en las edades más jóvenes, tanto mayor será el número de años de esperanza de vida que los argentinos pierdan. Esta situación hipotética se puede aplicar al análisis de la mortalidad general o de cualquier causa de muerte particular de interés ${ }^{2}$.

Finalmente, se plantearon diferentes escenarios futuros en relación al cambio de la mortalidad por atropellos y colisiones viales en los próximos 10 años. A tal efecto, se analizaron posibles modificaciones de las tasas de mortalidad específicas por edad en el espacio vial. La comparación entre la esperanza de vida observada en el trienio 20092011 y la obtenida de situaciones hipotéticas podría arrojar indicios para la planificación futura de los recursos económicos destinados a combatir la mortalidad vial en Argentina.

Los registros de la población segmentada por edad y sexo fueron tomados de: a) los dos últimos censos nacionales de población, hogares y viviendas, correspondientes a los años 2001 y 2010, y b) las proyecciones de población calculadas para el período 2000-2011 excepto para los años censales-, publicadas por la Dirección de Estadísticas Poblacionales.

\footnotetext{
2 Tal como sugiere Arriaga (2011), el límite de edad adoptado fue de 80 años, por dos razones: 1) la calidad de la información sobre mortalidad en personas de edades muy avanzadas puede presentar errores considerables, y 2) el número de años de esperanza de vida perdidos es relativamente bajo en personas que superan los 80 años de edad.
} 
La información sobre las causas de muerte, sexo y edad de los fallecidos se obtuvo de las bases de datos de la Dirección de Estadísticas e Investigación en Salud (DEIS), dependiente del Ministerio de Salud de la Nación. Los datos resultan de la recopilación que realizan los registros civiles, y constituyen una fuente de información secundaria.

Los decesos viales se identificaron según la décima revisión de la Clasificación Internacional de Enfermedades (OPS, 1995), mediante los códigos comprendidos entre V01 y V89. Estos códigos se refieren principalmente a aquellas muertes en las que interviene al menos un vehículo de motor y, en algunas ocasiones, los trenes. La inclusión del transporte por ferrocarriles resulta pertinente para la investigación, debido a que los trenes forman parte del sistema de transporte terrestre que utilizan los usuarios de la vía pública en el país. Además, se consideraron las defunciones como consecuencia de las secuelas de accidentes en vehículos a motor correspondientes al código Y850.

Los registros de las estadísticas vitales utilizados en este trabajo representan una de las perspectivas más conservadoras de la problemática vial, puesto que los datos de mortalidad provenientes de otras fuentes de información, como el Instituto de Seguridad y Educación Vial (ISEV) y la organización no gubernamental Luchemos por la Vida, le otorgan una magnitud aún mayor. Según información provista por esta asociación civil, en Argentina murieron en promedio 7.141 personas en el primer trienio analizado, cifra que supera en un $83 \%$ los registros oficiales. Para el segundo trienio la brecha se redujo a un $55 \%$, pues se registraron 7.687 decesos en promedio.

Estas divergencias en las cifras registradas por distintas instituciones y organismos se deben principalmente a la diferencia en la modalidad de registro de las defunciones. Las estadísticas vitales contabilizadas por el Ministerio de Salud solo consideran los fallecimientos ocurridos en el momento del evento vial, mientras que las cifras más elevadas fueron ajustadas por un factor de corrección -mediante un criterio aceptado por la OMS-, con la adición de la cantidad de muertos hasta 30 días posteriores al evento vial.

\section{Resultados}

Las muertes ocurridas por lesiones en eventos de tránsito terrestre en Argentina tuvieron un comportamiento variado en la primera década del siglo XXI. Como se muestra en el Gráfico 1, en los años 2000 y 2001 murieron por esta causa alrededor de 11 personas por cada 100.000. Para el año 2003 el riesgo de muerte por esta causa se redujo a 9,3, y se mantuvo por debajo de 10 hasta 2005. Luego de este último período, la tasa de mortalidad creció hasta igualar la cifra de 2001. Este indicador de riesgo de muerte creció más del $18 \%$ en 11 años, lo que resulta coherente con lo expresado en estudios realizados por la OMS, que indican un incremento de las muertes por esta causa. 
GRÁFICO 1

Tasa de mortalidad por atropellos y colisiones viales, según sexo

Argentina - 2000-2011

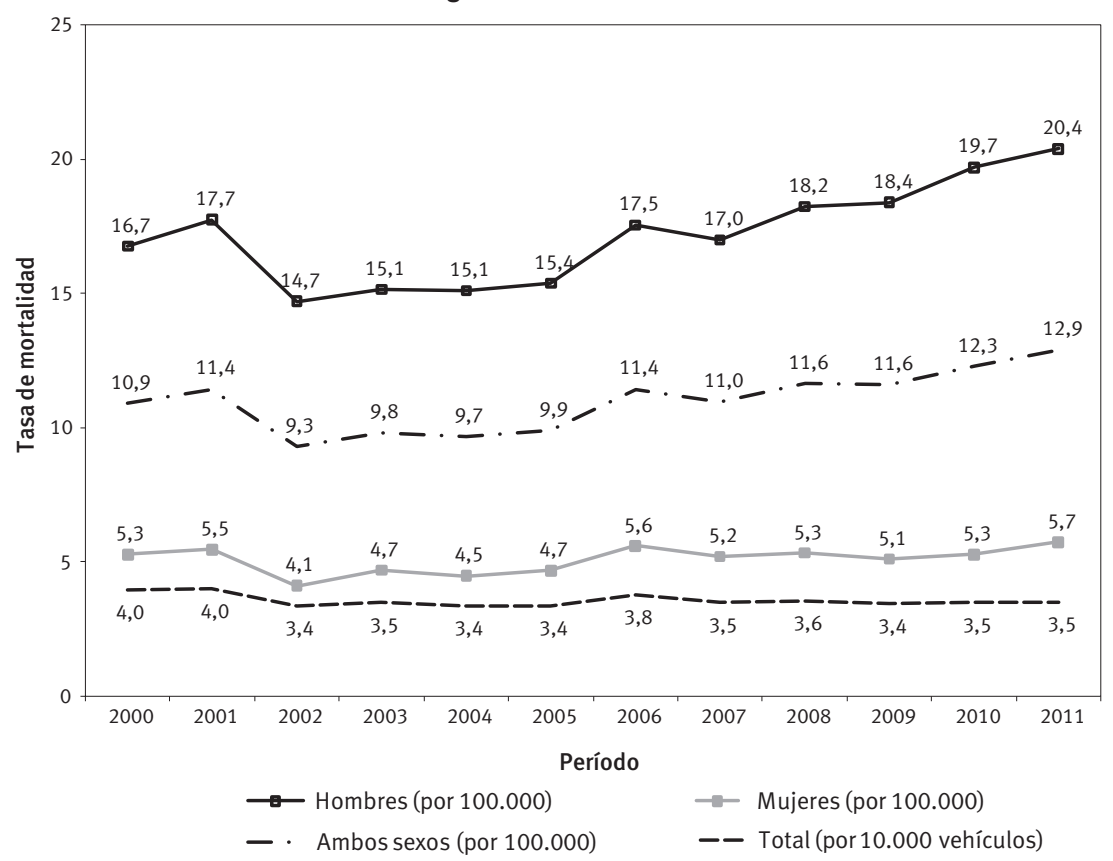

Fuente: Ministerio de Salud de Argentina, Dirección Nacional de Estadísticas e Información en Salud. Estadísticas Vitales. Buenos Aires: INDEC.

A pesar de su crecimiento, en especial durante la segunda mitad del decenio analizado, estas cifras resultaron significativamente menores que la tasa de mortalidad por colisiones viales mundiales, que fue estimada por la OMS en 18,8 muertos cada 100.000 habitantes. Incluso se puede observar que el país se caracteriza por un menor riesgo de muerte que la región de las Américas, cuya tasa de mortalidad vial es de 16,1. Si bien según la clasificación de la OMS Argentina es considerado un país de ingresos medios, su tasa de mortalidad por colisiones viales es menor que la de estos países (19,5 muertes) y superior a las mediciones de aquellos de ingresos altos (10,3) (OMS, 2009).

A diferencia de las variaciones observadas en la tasa de mortalidad por habitantes, la evolución del riesgo de muerte en función de la cantidad de vehículos registrados en el país muestra un comportamiento más homogéneo a lo largo de la década estudiada. En el año 2000 murieron 4 personas por cada 10.000 vehículos, mientras que 11 años después la cifra se redujo a 3,5 decesos. Si bien la tasa de mortalidad por colisiones viales cada 10.000 vehículos experimentó una leve reducción entre 2000 y 2011, el parque automotor argentino creció un 48,1\%, alcanzando los 15.025.245 vehículos registrados. En consecuencia, la tasa de motorización (número de vehículos por cada 1.000 habitantes) pasó de 274 en 2000 a 367 en 2011, lo que representó un crecimiento del 34,1\%. En este sentido, el parque automotor aumentó cuatro veces más que la población residente en el país. Este 
punto de vista pone de manifiesto que no hay evidencias de una relación directa entre la cantidad de vehículos que circularon en el territorio nacional en la primera década del siglo XXI y el riesgo de muerte de las personas.

Estos datos resultan coherentes con lo expresado en el informe de la OMS, en el que se señala que existe un mayor número de muertes por esta causa en los países de ingresos bajos y medios, que solo concentran el $48 \%$ del parque automotor registrado en el mundo (OMS, 2009).

Ahora bien, dado que el riesgo de muerte en colisiones viales presenta marcadas diferencias según la composición por sexo y edad de la población, el próximo apartado se ocupa del análisis de la mortalidad diferencial.

\section{Las tasas de mortalidad vial y su relación con el sexo y la edad}

La tasa de mortalidad vial masculina fue tres veces mayor que la femenina en los primeros dos años de la década analizada. En 2002 hubo una reducción de las tasas de ambos sexos, aunque su relación se incrementó a 3,6. Luego, entre 2003 y 2006, la sobremortalidad masculina mantuvo los niveles de los dos primeros años de la década. En el período comprendido entre 2007 y 2010 las tasas masculinas crecieron más que las femeninas y, en consecuencia, la sobremortalidad de los hombres se fue incrementando anualmente, hasta alcanzar un máximo de 3,7 muertes en 2010. En 2011 se registraron aumentos de las tasas de ambos sexos, pero la diferencia entre ellas se redujo levemente. En este sentido, es posible afirmar que en Argentina la población masculina enfrenta un mayor riesgo de morir por atropellos y colisiones viales que la femenina.

Las diferencias de las tasas de mortalidad vial entre hombres y mujeres se registran en todos los grupos etarios, aunque de manera más marcada en algunos. Como puede observarse en el Gráfico 2, en el trienio 2000-2002 tanto los varones como las mujeres menores de un año de edad se enfrentaron a riesgos de muerte similares por colisiones viales. Sin embargo, desde las edades tempranas (de 1 a 4 años) estas tasas comenzaron a diferenciarse, alcanzando una sobremortalidad de 2 hombres por cada mujer en el grupo de 5 a 14 años de edad. El contraste se incrementó a medida que aumentó la edad de los fallecidos, hasta alcanzar su valor máximo en el grupo de 20 a 24 años, en el que el riesgo para los hombres fue 4,6 veces mayor que para las mujeres. La divergencia se mantuvo relativamente constante hasta los 59 años, edad a partir de la cual las discrepancias en las tasas comenzaron a reducirse. Entre los 65 años y las edades más avanzadas murieron unos 3 hombres por cada mujer.

En el trienio 2009-2011 la relación entre las tasas de mortalidad por atropellos y colisiones viales de hombres y mujeres tuvo un comportamiento similar al del primer trienio de la década. Sin embargo, la mayor diferencia en el riesgo de muerte se registró en el grupo de 25 a 29 años, en el que los hombres enfrentaron un riesgo 6,2 veces mayor de morir que las mujeres. 
GRÁFICO 2

Sobremortalidad masculina general y por atropellos y colisiones, según grupos de edad Argentina - 2000-2011

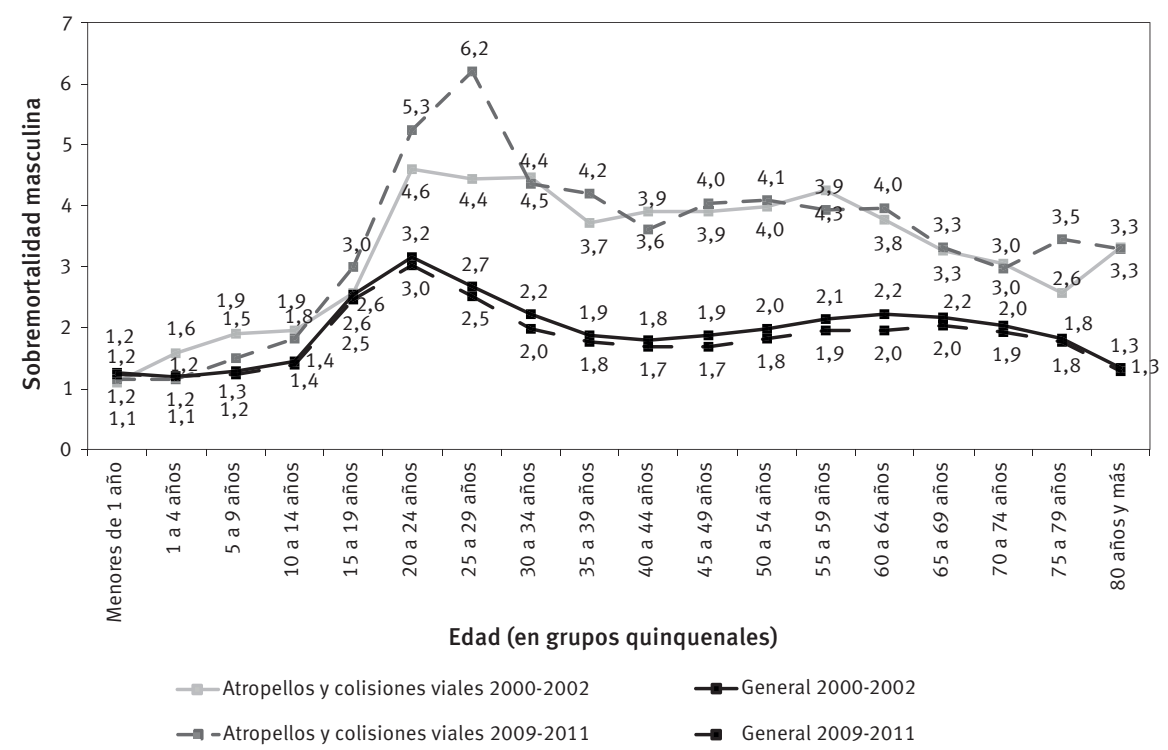

Fuente: Ministerio de Salud de Argentina, Dirección Nacional de Estadísticas e Información en Salud. Estadísticas Vitales. Buenos Aires: INDEC.

En ambos trienios, y en la mayoría de los grupos etarios, es posible observar que la sobremortalidad masculina por atropellos y colisiones viales fue mayor que el mismo indicador en el caso de la mortalidad general. Las principales diferencias se observaron entre los fallecidos de 20 a 64 años de edad. Las muertes ocurridas en el espacio vial representan una de las principales causas de fallecimiento que contribuye a incrementar la mortalidad diferencial entre ambos sexos.

Como puede observarse en el Gráfico 3, los niveles de riesgo de muerte por lesiones en colisiones viales en los primeros 14 años de vida de los argentinos fueron bajos, tanto en el trienio inicial como en el final de la década. En el trienio 2000-2002 la tasa de mortalidad masculina de los jóvenes de 20 a 24 años alcanzó los 25 decesos cada 100.000 varones, lo que representó uno de los riesgos más altos de todos los grupos etarios, solo superado por el de dos segmentos de edad: los hombres de 65 a 69 años y los mayores de 80 años.

Por su parte, para el período 2009-2011 es posible observar que el riesgo de muerte por colisiones viales se redujo levemente en las edades menores de 14 años. Por el contrario, entre los mayores de 15 años la tasa de mortalidad se incrementó, salvo en algunos grupos específicos (el de 40 a 44 años y los mayores de 80 años).

En tanto, el análisis del riesgo de muerte femenino en el trienio 2000-2002 pone en evidencia tres situaciones: en primer lugar la del grupo de 0 a 14 años, con tasas inferiores a 3 decesos cada 100.000 mujeres; en segundo lugar, la de las personas de entre 15 y 64 años de edad, con tasas cercanas a las 5,6 muertes, y finalmente la de las mujeres 
mayores de 65 años, con tasas de 7 fallecimientos o más. Los registros del trienio 20092011 revelan un comportamiento similar, aunque con pequeñas variaciones. La mayoría de los grupos etarios registraron tasas de mortalidad mayores, excepto las menores de 5 años, las niñas de 10 a 14 años, las mujeres de 25 a 29 años, 35 a 39 años y 75 a 79 años.

GRÁFICO 3

Tasa de mortalidad específica por atropellos y colisiones viales, según grupos de edad Argentina -2000-2011

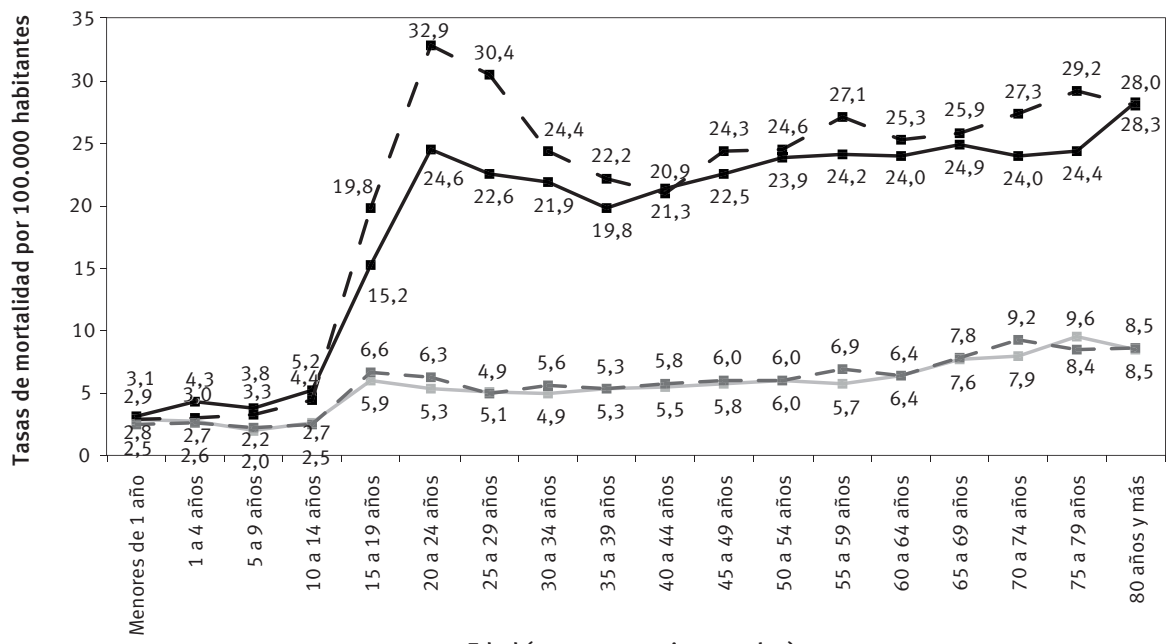

Edad (en grupos quinquenales)

$\rightarrow-$ Hombres 2000-2002 $\rightarrow$ Mujeres 2000-2002 $\rightarrow$ Hombres 2009-2011 $\rightarrow$-Mujeres 2009-2011

Fuente: Ministerio de Salud de Argentina, Dirección Nacional de Estadísticas e Información en Salud. Estadísticas Vitales. Buenos Aires: INDEC.

En el análisis de ambos sexos se puede observar que las tasas se incrementaron en algunos grupos etarios y se redujeron en otros, por lo que resulta pertinente complementar la observación con el indicador de los años de esperanza de vida perdidos, para evaluar el nivel y el cambio de la mortalidad.

En resumen, es posible apreciar que el riesgo de muerte fue más pronunciado entre los hombres que entre las mujeres, tanto en el trienio 2000-2002 como en el comprendido entre 2009 y 2011 . Además, las tasas de mortalidad masculinas registraron diferencias más elevadas que las femeninas al considerar los grupos quinquenales de edad.

\section{El impacto de la mortalidad vial en la esperanza de vida de los argentinos}

En el trienio 2000-2002 la población argentina perdió 18,9 años de esperanza de vida entre el nacimiento y los 80 años como consecuencia de los 284.760 fallecimientos promedio ocurridos en ese período, mientras que para el trienio 2009-2011 la muerte de 314.062 sujetos, en promedio, implicó la pérdida de 16,8 años de esperanza de vida en el mismo lapso vital. En efecto, después de nueve períodos anuales, los argentinos recu- 
peraron 2,1 años respecto del primer trienio analizado. Probablemente las mejoras de las condiciones de salud del país han provocado la menor pérdida de años de vida.

En relación a los atropellos y colisiones de tránsito, en el trienio 2000-2002 la población argentina perdió 0,6 años (siete meses) de esperanza de vida entre el nacimiento y los 80 años como consecuencia de las 3.902 muertes promedio por esta causa acontecidas en ese período, mientras que para el trienio 2009-2011 el deceso de 4.953 personas, en promedio, en el espacio vial, implicó la pérdida de 0,7 años (ocho meses) de esperanza de vida entre el nacimiento y los 80 años. El peso relativo del impacto de las muertes viales se incrementó entre ambos períodos del 3,1\% al 4\% en relación al total de años de esperanza de vida perdidos de la mortalidad general.

Los argentinos perdieron aproximadamente un mes adicional de esperanza de vida por la mortalidad vial respecto del primer trienio analizado. Entonces, si bien el saldo es positivo a causa de la menor pérdida de años de vida para el conjunto de las causas de muerte, también es cierto que la mortalidad por atropellos y colisiones viales resta tiempo de vida a la población nacional.

La mortalidad vial afectó de manera diferenciada a hombres y mujeres, y ellos fueron los que más años de esperanza de vida perdieron. En este sentido, la Tabla 1 muestra que las muertes masculinas en atropellos y colisiones viales provocaron la pérdida de más de 5 y 6 meses de esperanza de vida en los trienios 2000-2002 y 2009-2011, respectivamente, mientras que estas cifras se redujeron a menos de 2 meses de esperanza de vida de las mujeres en ambos trienios.

TABLA 1

Años de esperanza de vida perdidos, según sexo y causas de muerte Argentina - 2000-2011

\begin{tabular}{lcccc}
\hline \multicolumn{1}{c}{$\begin{array}{c}\text { Sexo y } \\
\text { causa de muerte }\end{array}$} & $\mathbf{2 0 0 0 - 2 0 0 2}$ & $\mathbf{2 0 0 9 - 2 0 1 1}$ & Cambio total (\%) & Cambio anual (\%) \\
\hline Hombres & $\mathbf{1 1 , 9 3}$ & $\mathbf{1 0 , 4 5}$ & $-\mathbf{0 , 1 2}$ & $-\mathbf{1 , 4 6}$ \\
Atropellos y colisiones viales & 0,45 & 0,52 & 0,15 & 1,61 \\
Resto de causas & 11,48 & 9,93 & $-0,14$ & $-1,60$ \\
Mujeres & $\mathbf{6 , 9 5}$ & $\mathbf{6 , 3 1}$ & $-\mathbf{0 , 0 9}$ & $-\mathbf{1 , 0 6}$ \\
Atropellos y colisiones viales & 0,14 & 0,14 & 0,06 & 0,68 \\
Resto de causas & 6,81 & 6,17 & $-0,09$ & $-1,09$ \\
Total & $\mathbf{1 8 , 8 8}$ & $\mathbf{1 6 , 7 6}$ & $-\mathbf{0 , 1 1}$ & $-\mathbf{1 , 3 1}$ \\
\hline
\end{tabular}

Fuente: Ministerio de Salud de Argentina, Dirección Nacional de Estadísticas e Información en Salud. Estadísticas Vitales. Buenos Aires: INDEC.

El hecho de que la mortalidad impacte anticipadamente a los hombres podría traer aparejado un conjunto de cambios en los roles tradicionales que han desempeñado las personas de cada sexo. Así, por ejemplo, la muerte de un hombre que ocupaba el rol de jefe de hogar antes de su deceso podría implicar la desarticulación de la estructura familiar original y dar paso a un hogar monoparental. En este sentido, quien quedaría a cargo de las responsabilidades familiares, la mujer, debería adoptar nuevos comportamientos, ac- 
tividades y responsabilidades que antes cumplía el hombre (protección familiar y principal sostén económico del hogar, entre otras) (MARTíNEZ; MERLINO; ESCANÉS, 2011).

Una observación de los grupos etarios más afectados por este tipo de muertes muestra que los varones de 20 a 24 años son quienes generan mayor impacto en la esperanza de vida, en ambos trienios. Como puede verse en la Tabla 2, en el período 2000-2002 el grupo de hombres de 15 a 44 años representó el 64\% de los años de esperanza de vida perdidos por atropellos y colisiones viales, mientras que esa cifra ascendió al $68 \%$ para el segundo trienio.

En el caso de las mujeres, se observa que en ambos trienios las de 15 a 29 años fueron las que experimentaron mayores pérdidas de años de esperanza de vida. Esto se hace evidente con mayor intensidad en el grupo de 20 a 24 años de edad, que perdió poco más de 5 y 6 días de esperanza de vida en los trienios 2000-2002 y 2009-2011, respectivamente.

TABLA 2

Años de esperanza de vida perdidos por atropellos y colisiones viales, según sexo y edad

Argentina - 2000-2011

\begin{tabular}{lccccc}
\hline \multirow{2}{*}{ Grupos etarios } & \multicolumn{2}{c}{ Hombres } & & \multicolumn{2}{c}{ Mujeres } \\
\cline { 2 - 3 } \cline { 5 - 6 } 0 a 14 años & $\mathbf{2 0 0 0 - 2 0 0 2}$ & $\mathbf{2 0 0 9 - 2 0 1 1}$ & & $\mathbf{2 0 0 0 - 2 0 0 2}$ & $\mathbf{2 0 0 9 - 2 0 1 1}$ \\
15 a 29 años & 0,046 & 0,038 & & 0,026 & 0,026 \\
30 a 44 años & 0,156 & 0,207 & & 0,043 & 0,048 \\
45 a 59 años & 0,131 & 0,145 & & 0,032 & 0,034 \\
60 años y más & 0,084 & 0,094 & & 0,023 & 0,024 \\
Total & 0,032 & 0,037 & & 0,012 & 0,013 \\
\hline
\end{tabular}

Fuente: Ministerio de Salud de Argentina, Dirección Nacional de Estadísticas e Información en Salud. Estadísticas Vitales. Buenos Aires: INDEC.

La mortalidad por atropellos y colisiones de tránsito tiene mayor impacto en la pérdida de años de esperanza de vida en las edades comprendidas entre los 15 y los 44 años. De este modo, por ejemplo, el fallecimiento de una persona de 20 años por lesiones de tránsito reduciría la capacidad de la sociedad para reproducirse (tenencia de hijos), para recibir educación formal en la universidad y para insertarse en el ámbito laboral, entre otros aspectos.

\section{El impacto de posibles cambios en las tasas de mortalidad vial}

En este apartado se plantean cuatro escenarios hipotéticos, claramente diferenciados, respecto de la evolución de la mortalidad vial, aplicados en igual magnitud a hombres y mujeres de todos los grupos etarios, con la finalidad de poner en evidencia el impacto diferencial que tendría una variación constante de este indicador en todos los subgrupos de la población. En primer lugar, se consideraron dos escenarios vinculados a posibles incrementos de las tasas. En segundo lugar, y en contraste, se imaginaron dos contextos 
que se sustentan en una hipótesis optimista, vinculada a la reducción del riesgo de muerte por lesiones en el tránsito.

En el primer supuesto se propone un posible incremento de las tasas de mortalidad vial de un $50 \%$ para cada sexo y grupo etario en el próximo decenio. La idea de analizar este escenario futuro e hipotético sobre el riesgo de muerte encuentra su fundamento en las publicaciones de la OMS, que estiman que para el año 2020 las muertes por esta causa se incrementarían de 1,27 a 1,9 millones anuales (OMS, 2013). En este sentido, se esperaría que la mortalidad vial aumente en poco más del $50 \%$ respecto de las cifras del trienio 2009-2011.

En otro de los escenarios se reflexiona sobre una segunda alternativa pesimista, aunque en menor medida que en el primer supuesto. A partir de esa base, se analiza de qué manera impactaría sobre la esperanza de vida de los argentinos un incremento del $15 \%$ de las tasas de mortalidad vial para cada sexo y grupo etario. Este escenario hipotético surge de la tendencia nacional del primer decenio del siglo XXI, cuando la mortalidad por atropellos y colisiones de tránsito aumentó un 14,7\% entre 2000-2002 y 2009-2011.

En contrapartida, se plantean dos hipótesis optimistas. En estos casos, se analiza cuánto se incrementaría la expectativa de vida de los argentinos si el riesgo de muerte en el espacio vial se redujera un 15\% (supuesto 3) y un 50\% (supuesto 4) para el año 2020.

Los resultados indican que si las tasas de mortalidad vial se incrementaran un $50 \%$ para 2020, la esperanza de vida al nacer de los hombres se reduciría en aproximadamente 3 meses, o cerca de un mes si se cumpliera el segundo supuesto (aumento del 15\%). Ambos escenarios, con mayor o menor impacto sobre la expectativa de vida, plantean una visión pesimista respecto de la evolución del riesgo de muerte por lesiones de tránsito y su relación con el número de años que se espera que viva la población argentina.

En contraste, si la evolución de las tasas se aproximara a la situación planteada en el tercer supuesto (reducción del 15\%), la esperanza de vida masculina aumentaría a 72,2 años, mientras que alcanzaría los 72,4 años de concretarse una reducción del $50 \%$ de las tasas de mortalidad vial.

Respecto de la situación de las mujeres, un incremento del $50 \%$ de las tasas de mortalidad vial implicaría la reducción de aproximadamente un mes en la esperanza de vida al nacer. En otros términos, las mujeres perderían la tercera parte del descenso de los hombres. En contrapartida, si en el año 2020 se redujeran las tasas en esa misma proporción (supuesto 4), las mujeres sumarían un mes a la expectativa de vida. Por su parte, la variación del $15 \%$ en el riesgo de muerte, tanto por defecto como por exceso, propuesta en los escenarios intermedios, tendría impactos menores sobre la esperanza de vida de las mujeres argentinas (aproximadamente entre 7 y 10 días).

El impacto de estas transiciones hipotéticas sería mayor entre los hombres que entre las mujeres, dado que ellos pierden más años de vida. Sin embargo, si no se adoptan medidas tendientes a reducir el impacto de la mortalidad de ambos sexos, producida como conse- 
cuencia de lesiones por atropellos y colisiones viales, es probable que el riesgo de muerte vial en Argentina se ubique entre la primera dupla de escenarios posibles propuestos.

\section{Discusión}

En primer lugar, mediante el análisis propuesto es posible destacar que hubo un incremento de las muertes ocurridas en el espacio vial en ocasión del tránsito en Argentina. El número de fallecimientos viales creció un 27\% entre los trienios 2000-2002 y 2009-2011, lo que implicó un incremento del riesgo de muerte en función de la población expuesta, que pasó de 10,8 a 12,3 decesos cada 100.000 habitantes (aproximadamente un 15\%). El sentido ascendente de los registros de víctimas mortales en el primer decenio del siglo XXI se produce como consecuencia de una multiplicidad de factores. En este sentido, es probable que la tendencia en alza de la mortalidad vial se corresponda con ciertas actitudes, vínculos interpersonales, prácticas de desplazamiento en las ciudades, disociaciones entre normas formales y conductas individuales y ausencia de controles del Estado.

En segundo lugar, este trabajo pone de manifiesto la relación entre las tasas de mortalidad vial y el sexo de las personas fallecidas. Los hombres resultaron más afectados que las mujeres por este tipo de decesos, tal como lo refleja el patrón de mortalidad en la mayoría de los países del mundo. En este sentido, en el trienio 2000-2002 los fallecimientos femeninos por atropellos y colisiones viales representaron un tercio de los masculinos. Para el trienio 2009-2011 la sobremortalidad masculina registró cerca de 4 muertes de varones por cada deceso de una mujer.

En tercer lugar, este trabajo destaca la diferencia del riesgo de muerte entre los grupos quinquenales de edad analizados. En el primer trienio estudiado, las tasas de mortalidad vial mostraron niveles similares para ambos sexos entre el nacimiento y los 14 años de edad (menores a 5 decesos cada 100.000 habitantes), aunque levemente superiores para los varones. La diferenciación entre hombres y mujeres se volvió más acentuada en el grupo de 15 a 19 años (superior a 15 decesos cada 100.000 habitantes), alcanzando un valor máximo entre los 20 y los 24 años de edad (superior a 24 decesos cada 100.000 habitantes), como consecuencia del incremento del riesgo de muerte que enfrentaron los hombres. Desde las edades jóvenes, cercanas a los 25 años, hasta el final de la vida, las tasas de mortalidad específicas por edad se mantuvieron relativamente estables en los niveles mencionados para el último grupo quinquenal de ambos sexos. Para el segundo trienio analizado, el riesgo de muerte se incrementó en los hombres de la mayoría de los grupos etarios, mientras que para las mujeres no hubo diferencias significativas respecto de 2000-2002. En consecuencia, la sobremortalidad masculina entre los 20 y los 24 años de edad aumentó a 6 fallecimientos.

En lo que respecta al indicador de los años de esperanza de vida perdidos, la mortalidad impactó anticipadamente a los hombres. Así, en 2000-2002 ellos perdieron alrededor del $77 \%$ de los años de esperanza de vida por este tipo de fallecimientos, lo que representó 
0,45 años de esperanza de vida perdidos, mientas que las mujeres perdieron 0,14, un tercio del mismo indicador para los varones. En 2009-2011 se mantuvo el patrón del primer trienio, aunque se profundizó levemente la diferencia entre varones y mujeres debido al incremento de los años de esperanza de vida perdidos entre los primeros.

En términos generales, esta investigación pone de manifiesto la necesidad de mejorar las condiciones de salud de los argentinos en este aspecto. Si bien se han implementado políticas públicas en el marco del Plan Nacional de Seguridad Vial, han resultado insuficientes para lograr una reducción de la mortalidad por esta causa. Por lo tanto, es necesario diseñar y ejecutar eficazmente políticas en beneficio de la seguridad vial, para evitar que sigan incrementándose las lesiones, las muertes y los costos económicos relacionados a este tipo de eventos. En este sentido, resulta fundamental que se adopten en el país medidas de educación vial, fiscalización y control del cumplimiento de las normas de tránsito, y que se invierta en el mejoramiento de la infraestructura vial, entre otros aspectos. De esta manera, se podría evitar que en el próximo decenio se incremente la proporción de actores sociales - peatones, ciclistas, motociclistas y conductores de vehículos a motor- afectados por la problemática vial, a causa del aumento de la desarticulación de las unidades familiares, la profundización de las secuelas físicas y psíquicas y la modificación abrupta del plan de vida por decesos ocurridos en atropellos o colisiones de tránsito.

\section{Referencias}

AGENCIA NACIONAL DE SEGURIDAD VIAL. Educación vial. Convivir en el espacio público. Buenos Aires, s/d. Disponible en: 〈http://www.educacionvial.gov.ar/pdf/material/secundario/conviviren-el-espacio-publico-c.pdf> Acceso en: 25 jun. 2013.

Relevamiento de indicadores estadísticos en materia de Seguridad Vial en Argentina, consignando autor y entes que utilizan cada uno. Buenos Aires. 2010. Disponible en: 〈http://observatoriovial.seguridadvial.gov.ar/documentos/ops/relevamiento-de-indicadoresestadisticos-en-materia-de-seguridad-vial-en-argentina.pdf〉. Acceso en: 27 ago. 2012.

ARRIAGA, E. Los años de vida perdidos: su utilización para medir los niveles y cambios de la mortalidad. Notas de Población, v. 63, p. 7-38, 1996.

. Análisis demográfico de la mortalidad. Córdoba: Centro de Investigaciones y Estudios sobre Cultura y Sociedad - CONICET - UNC, 2011.

BELTRAMINO, J. C.; CARRERA, E. El respeto a las normas de tránsito en la ciudad de Santa Fe, Argentina. Revista Panamericana Salud Pública, v. 22, n. 2, p. 141-145, 2007.

CHANDRAN, A.; KAHN, G.; SOUSA, T.; PECHANSKY, F.; BISHAI, D. M.; HYDER, A. A. Impact of road traffic deaths on expected years of life lost and reduction in life expectancy in Brazil. Demography, n. 50, p. 229-236, 2013.

CENTRO LATINOAMERICANO Y CARIBEÑO DE DEMOgRAFíA. Demografía I. México, D.F.: PROLAPIISUNAMS, 1997.

COMISIÓN NACIONAL DE PROGRAMAS DE INVESTIGACIÓN SANITARIA. Aspectos demográficos y sociales de los accidentes de tránsito en áreas seleccionadas de la Argentina. Buenos Aires: Ministerio de Salud y Ambiente de la Nación, 2006. 
DEFENSOR DEL PUEBLO DE LA NACIÓN. Informe especial sobre seguridad vial en Argentina. Buenos Aires, 2005. Disponible en: 〈http://www.lapampa.edu.ar/educacionvial/Documentos/03_ Documentos_de_Consulta/DPN_Seguridad_Vial_2005.pdf>. Acceso en: 8 ago. 2012.

FLEITAS, D. Accidentes de tránsito en Argentina. Segundo Informe / 1997-2008. Buenos Aires: Asociación para Políticas Públicas, 2010. Disponible en: 〈http://www.app.org.ar/wp-content/ uploads/2011/04/Accidentes-de-Transito-en-Argentina-2010-final.pdf>. Acceso en: 15 jun. 2013.

FOSCHIATTI, A. M.; LUCCA, A.; RAMÍREZ, L.; REY, C.; CARDOZO, O.; SOLARI, M. El impacto social de los accidentes de tránsito y su relación con los servicios hospitalarios, en Resistencia. Resistencia, s/d. Disponible en: 〈http://www.unne.edu.ar/unnevieja/Web/cyt/cyt/2001/2Humanisticas/H-007.pdf〉. Acceso en: 12 feb. 2013.

GARCETTE, N. Seguridad vial en la Argentina. Buenos Aires: Observatorio Social, marzo 2010 (Serie Informes de Coyuntura, 13). Disponible en: 〈http://observatoriosocial.com.ar/pdfs/ informe_13.pdf〉. Acceso en: 15 mar. 2013.

INSTITUTO DE SEGURIDAD Y EDUCACIÓN VIAL. Análisis de la siniestralidad vial Argentina 2012/2011. Buenos Aires, 2012. Disponible en: 〈http://www.isev.com.ar/isev_home/materiales/ analisis_de_siniestralidad_2012-2011.pdf〉. Acceso en: 2 jun. 2013.

INTERNATIONAL TRAFFIC SAFETY. Road safety annual report 2011. París, 2011. Disponible en: 〈http://www.internationaltransportforum.org/irtadpublic/pdf/11/rtadReport.pdf〉. Acceso en: 7 ago. 2013.

LAPOSTOLLE, A.; GADEGBEKU, B.; NDIAYE, A.; AMOROS, E.; CHIRON, M.; SPIRA, A.; LAUMON, B. The burden of road traffic accidents in a French Department: the description of the injuries and recent changes. BMC Public Health, v. 9, n. 386, 2009.

MACÍAS, G. R.; ALMEIDA FILHO, N.; MARCIO, A. Análisis de las muertes por accidentes de tránsito en el municipio de Lanús, Argentina, 1998-2004. Salud Colectiva, v. 6, n. 3, p. 313-328, 2010.

MARTÍNEZ, A.; MERLINO, A; ESCANÉS, G. Representaciones sociales de la masculinidad y agresividad en el tránsito. La ira al conducir en Argentina. Barbarói, v. 35, n. 2, p. 199-217, 2011.

MERLINO, A. De la argumentación a los modelos de acción/situación en el discurso sobre el tráfico vehicular en Argentina. Discurso y Sociedad, v. 4, n. 2, p. 257-293, 2010.

NAZIF, J. I.; PÉREZ SALAS, G. Siniestralidad vial en América Latina y el Caribe: desempeño reciente y desafíos futuros. Boletín FAL, n. 6, p. 1-8, 2013.

OMS - Organización Mundial de la Salud. Informe mundial sobre prevención de los traumatismos causados por el tránsito: resumen. Ginebra, 2004. Ginebra, 2009.

Informe sobre la situación mundial de la seguridad vial: es hora de pasar a la acción.

. Global status report on road safety 2013: supporting a decade of action. Ginebra, 2013.

OPS - Organización Panamericana de la Salud. Clasificación Estadística Internacional de Enfermedades y Problemas Relacionados con la Salud. 10 a revisión. Washington, D.C., 1995.

D.C., 2009.

. Informe sobre el estado de la seguridad vial en la región de las Américas. Washington,

Resumen ejecutivo, estado de conocimiento y agenda de prioridades para la toma de decisiones en seguridad vial, en Argentina. Buenos Aires, 2007.

PEDEN, M.; TOROYAN, T. Road traffic injuries in South Asia: national and organisational policy responses. Journal of the College of Physicians and Surgeons-Pakistan, n. 14, p. 722-725, 2004. 
PELTZER, R. Accidentes de tránsito: teoría de la atribución, locus de control y adopción de medidas preventivas. Mar del Plata, 2003. Mimeo.

POLINDER, S.; MEERDING, W. J.; MULDER, S.; PETRIDOU, E.; VAN BEECK, E.; EUROCOST REFERENCE GROUP. Assessing the burden of injury in six European countries. Bulletin of the World Health Organization, v. 8, n. 5, p. 27-34, 2007.

PRADO, T.; MUÑOZ DE LA ROSA, D. Politraumatismo: accidentes de tránsito. Revista de la Asociación Argentina de Ortopedia y Traumatología, v. 74, n. 1, p. 6-12, 2009.

REY, C. E. Las condiciones ambientales de la vida humana. El tránsito como generador de riesgo de accidentes en la ciudad de Resistencia. Tesis (Maestría en Gestión Ambiental y Ecología). Facultad de Arquitectura y Urbanismo. Universidad Nacional del Nordeste, Resistencia, 1999.

TOLEDO, R. Sociología del tránsito. Buenos Aires: Colegio de Sociólogos de la Provincia de Buenos Aires, 2007.

\section{Sobre el autor}

Gabriel Escanés es demógrafo, magíster en Demografía por la Universidad Nacional de Córdoba. Se desempeña como becario en el Centro de Investigaciones y Estudios sobre Cultura y Sociedad (CIECS-CONICET).

\section{Dirección postal}

Manuel Freire 3281, CP X5002AXA, Córdoba, Argentina

\section{Resumo}

Tendências da mortalidade por atropelamentos e colisões de trânsito na Argentina entre 2000-2002 e 2009-2011

$\mathrm{O}$ artigo analisa a mortalidade viária, com o objetivo de medir o impacto que tiveram as mortes por atropelamentos e colisões de trânsito na esperança de vida dos argentinos, entre os triênios 2000-2002 e 2009-2011. Foi utilizado o índice de anos de vida perdidos, que serviu para estabelecer o número de anos que deveriam ter vivido as pessoas que morreram devido a lesões causadas ao transitar nas vias públicas, seja como pedestres, ciclistas, motociclistas ou ocupantes de veículos a motor. Os registros da população por idade e sexo foram obtidos dos dois últimos censos nacionais correspondentes a 2001 e 2010. As informações sobre as causas de morte, sexo e idade dos falecidos foram obtidas a partir dos bancos de dados do Dirección de Estadísticas e Información en Salud (DEIS). Os principais resultados indicam que, no período 2000-2002, a população Argentina perdeu 0,59 ano (sete meses) de esperança de vida - entre o nascimento e os 80 anos - como consequência de resultado de mortes no espaço viário, enquanto este número alcançou uma perda de 0,66 ano (oito meses) para o triênio 2009-2011.

Palavras-chave: Colisões. Trânsito. Atropelamento. Esperança de vida. Mortalidade. 


\begin{abstract}
Mortality trends by run-over accidents and traffic collisions in Argentina between 2000-2002 and 2009-2011

Road mortality was analyzed with the aim to measure the impact that deaths by run-over accidents and traffic collisions had on Argentineans' life expectancy, comparing the 2000-2002 and the 2009-2011 triennia. The index of lost years of life expectancy was used, which made it possible to establish the number of years that people would have lived if they had not died in accidents, either as pedestrians, cyclists, motorcyclists or occupants of motor vehicles. Information of population by sex and age were obtained from the two past national population censuses of 2001 and 2010. Information on causes of death, by sex and age of the deceased were obtained from Argentina's Statistical Office and Health Information (DEIS). The main results indicate that in 2000-2002 period, the Argentine population lost 0.59 years (seven months) of life expectancy - from birth to the age of 80 - as a consequence of deaths on the roads, while this number suffered a loss of 0.66 years (eight months) for the 2009-2011 period.
\end{abstract}

Keywords: Collisions. Traffic. Run-over accidents. Life expectancy. Mortality.

Recebido para publicação em 20/09/2013 Aceito para publicação em 24/05/2015 


\section{Anexo}

\begin{tabular}{|c|c|c|c|c|c|c|c|c|c|c|}
\hline \multirow{2}{*}{$\begin{array}{c}\text { Edad } \\
\quad \mathbf{x}\end{array}$} & \multicolumn{10}{|c|}{ Argentina, tabla de mortalidad de hombres } \\
\hline & $\mathrm{n}$ & $\mathrm{nMx}$ & nax & nqx & lx & $n d x$ & $\mathrm{nLx}$ & $5 \mathrm{Px}$ & Tx & ex \\
\hline \multicolumn{11}{|c|}{ Trienio 2000-2002 } \\
\hline 0 & 1 & 0,018 & 0,094 & 0,018 & 100000 & 1783,4 & 98383,8 & 0,981 & 7027363,4 & 70,27 \\
\hline 1 & 4 & 0,001 & 1,599 & 0,003 & 98216,6 & 294,8 & 392158,4 & 0,997 & 6928979,6 & 70,55 \\
\hline 5 & 5 & 0,000 & 2,500 & 0,001 & 97921,7 & 144,8 & 489246,5 & 0,998 & 6536821,2 & 66,76 \\
\hline 10 & 5 & 0,000 & 2,500 & 0,002 & 97776,9 & 170,9 & 488457,1 & 0,997 & 6047574,6 & 61,85 \\
\hline 15 & 5 & 0,001 & 2,500 & 0,004 & 97606,0 & 426,1 & 486964,7 & 0,994 & 5559117,5 & 56,95 \\
\hline 20 & 5 & 0,001 & 2,500 & 0,007 & 97179,9 & 724,6 & 484087,9 & 0,992 & 5072152,8 & 52,19 \\
\hline 25 & 5 & 0,002 & 2,500 & 0,009 & 96455,3 & 864,2 & 480115,7 & 0,990 & 4588064,9 & 47,57 \\
\hline 30 & 5 & 0,002 & 2,500 & 0,010 & 95591,0 & 970,9 & 475527,9 & 0,989 & 4107949,2 & 42,97 \\
\hline 35 & 5 & 0,003 & 2,500 & 0,013 & 94620,1 & 1195,9 & 470110,8 & 0,985 & 3632421,3 & 38,39 \\
\hline 40 & 5 & 0,004 & 2,500 & 0,018 & 93424,2 & 1650,7 & 462994,4 & 0,978 & 3162310,5 & 33,85 \\
\hline 45 & 5 & 0,006 & 2,500 & 0,027 & 91773,5 & 2490,1 & 452642,5 & 0,965 & 2699316,1 & 29,41 \\
\hline 50 & 5 & 0,009 & 2,500 & 0,043 & 89283,4 & 3877,3 & 436724,1 & 0,945 & 2246673,6 & 25,16 \\
\hline 55 & 5 & 0,014 & 2,500 & 0,068 & 85406,2 & 5787,0 & 412563,5 & 0,915 & 1809949,5 & 21,19 \\
\hline 60 & 5 & 0,022 & 2,500 & 0,104 & 79619,2 & 8251,4 & 377467,6 & 0,874 & 1397386,0 & 17,55 \\
\hline 65 & 5 & 0,033 & 2,500 & 0,152 & 71367,8 & 10833,9 & 329754,4 & 0,818 & 1019918,3 & 14,29 \\
\hline 70 & 5 & 0,049 & 2,500 & 0,219 & 60533,9 & 13236,7 & 269577,8 & 0,742 & 690164,0 & 11,40 \\
\hline 75 & 5 & 0,073 & 2,500 & 0,309 & 47297,2 & 14594,2 & 200000,6 & 0,524 & 420586,2 & 8,89 \\
\hline 80 & + & 0,148 & 6,745 & 1,000 & 32703,0 & 32703,0 & 220585,6 & & 220585,6 & 6,75 \\
\hline \multicolumn{11}{|c|}{ Trienio 2009-2011 } \\
\hline 0 & 1 & 0,013 & 0,080 & 0,013 & 100000 & 1293,2 & 98809,9 & 0,986 & 7215619,0 & 72,16 \\
\hline 1 & 4 & 0,001 & 1,614 & 0,002 & 98706,8 & 230,7 & 394277,0 & 0,998 & 7116809,2 & 72,10 \\
\hline 5 & 5 & 0,000 & 2,500 & 0,001 & 98476,2 & 126,5 & 492064,6 & 0,999 & 6722532,2 & 68,27 \\
\hline 10 & 5 & 0,000 & 2,500 & 0,002 & 98349,7 & 163,7 & 491339,2 & 0,997 & 6230467,6 & 63,35 \\
\hline 15 & 5 & 0,001 & 2,500 & 0,004 & 98186,0 & 406,7 & 489913,3 & 0,994 & 5739128,4 & 58,45 \\
\hline 20 & 5 & 0,001 & 2,500 & 0,007 & 97779,3 & 676,0 & 487206,6 & 0,993 & 5249215,1 & 53,68 \\
\hline 25 & 5 & 0,002 & 2,500 & 0,008 & 97103,3 & 763,8 & 483607,0 & 0,992 & 4762008,5 & 49,04 \\
\hline 30 & 5 & 0,002 & 2,500 & 0,009 & 96339,5 & 827,5 & 479628,7 & 0,990 & 4278401,6 & 44,41 \\
\hline 35 & 5 & 0,002 & 2,500 & 0,011 & 95512,0 & 1011,6 & 475031,0 & 0,987 & 3798772,9 & 39,77 \\
\hline 40 & 5 & 0,003 & 2,500 & 0,015 & 94500,4 & 1402,9 & 468994,8 & 0,981 & 3323741,9 & 35,17 \\
\hline 45 & 5 & 0,005 & 2,500 & 0,023 & 93097,5 & 2127,9 & 460168,0 & 0,970 & 2854747,1 & 30,66 \\
\hline 50 & 5 & 0,007 & 2,500 & 0,036 & 90969,7 & 3308,2 & 446577,8 & 0,953 & 2394579,1 & 26,32 \\
\hline 55 & 5 & 0,012 & 2,500 & 0,058 & 87661,4 & 5065,2 & 425644,1 & 0,927 & 1948001,4 & 22,22 \\
\hline 60 & 5 & 0,019 & 2,500 & 0,089 & 82596,2 & 7373,1 & 394548,3 & 0,889 & 1522357,2 & 18,43 \\
\hline 65 & 5 & 0,029 & 2,500 & 0,134 & 75223,1 & 10075,3 & 350927,3 & 0,836 & 1127809,0 & 14,99 \\
\hline 70 & 5 & 0,044 & 2,500 & 0,199 & 65147,8 & 12937,9 & 293394,3 & 0,762 & 776881,7 & 11,92 \\
\hline 75 & 5 & 0,067 & 2,500 & 0,287 & 52209,9 & 14975,7 & 223610,4 & 0,538 & 483487,4 & 9,26 \\
\hline 80 & + & 0,143 & 6,980 & 1,000 & 37234,3 & 37234,3 & 259877,0 & & 259877,0 & 6,98 \\
\hline
\end{tabular}

Fuente: Ministerio de Salud de Argentina, Dirección Nacional de Estadísticas e Información en Salud. Estadísticas Vitales. Buenos Aires: INDEC. 


\begin{tabular}{|c|c|c|c|c|c|c|c|c|c|c|}
\hline \multirow{2}{*}{$\begin{array}{c}\text { Edad } \\
\mathbf{x} \\
\end{array}$} & \multicolumn{10}{|c|}{ Argentina, tabla de mortalidad de mujeres } \\
\hline & $\mathrm{n}$ & $\mathrm{nMx}$ & nax & nqx & lx & $\mathrm{ndx}$ & $\mathrm{nLx}$ & $5 \mathrm{Px}$ & Tx & ex \\
\hline \multicolumn{11}{|c|}{ Trienio 2000-2002 } \\
\hline 0 & 1 & 0,015 & 0,093 & 0,014 & 100000 & 1449,9 & 98685,7 & 0,985 & 7815677,3 & 78,16 \\
\hline 1 & 4 & 0,001 & 1,500 & 0,003 & 98550,1 & 249,1 & 393577,7 & 0,998 & 7716991,6 & 78,31 \\
\hline 5 & 5 & 0,000 & 2,500 & 0,001 & 98301,0 & 114,1 & 491219,7 & 0,999 & 7323414,0 & 74,50 \\
\hline 10 & 5 & 0,000 & 2,500 & 0,001 & 98186,9 & 119,5 & 490635,6 & 0,998 & 6832194,3 & 69,58 \\
\hline 15 & 5 & 0,000 & 2,500 & 0,002 & 98067,4 & 186,5 & 489870,5 & 0,998 & 6341558,7 & 64,67 \\
\hline 20 & 5 & 0,001 & 2,500 & 0,003 & 97880,8 & 259,1 & 488756,5 & 0,997 & 5851688,2 & 59,78 \\
\hline 25 & 5 & 0,001 & 2,500 & 0,003 & 97621,8 & 325,7 & 487294,7 & 0,996 & 5362931,7 & 54,94 \\
\hline 30 & 5 & 0,001 & 2,500 & 0,004 & 97296,1 & 437,6 & 485386,4 & 0,995 & 4875637,0 & 50,11 \\
\hline 35 & 5 & 0,001 & 2,500 & 0,006 & 96858,5 & 620,1 & 482742,0 & 0,992 & 4390250,6 & 45,33 \\
\hline 40 & 5 & 0,002 & 2,500 & 0,009 & 96238,4 & 910,5 & 478915,6 & 0,988 & 3907508,5 & 40,60 \\
\hline 45 & 5 & 0,003 & 2,500 & 0,014 & 95327,9 & 1363,6 & 473230,4 & 0,982 & 3428592,9 & 35,97 \\
\hline 50 & 5 & 0,004 & 2,500 & 0,022 & 93964,3 & 2035,9 & 464731,6 & 0,973 & 2955362,5 & 31,45 \\
\hline 55 & 5 & 0,007 & 2,500 & 0,032 & 91928,4 & 2957,8 & 452247,5 & 0,960 & 2490630,9 & 27,09 \\
\hline 60 & 5 & 0,010 & 2,500 & 0,048 & 88970,6 & 4296,9 & 434110,9 & 0,940 & 2038383,4 & 22,91 \\
\hline 65 & 5 & 0,015 & 2,500 & 0,073 & 84673,7 & 6158,0 & 407973,6 & 0,907 & 1604272,6 & 18,95 \\
\hline 70 & 5 & 0,024 & 2,500 & 0,114 & 78515,7 & 8940,9 & 370226,3 & 0,854 & 1196299,0 & 15,24 \\
\hline 75 & 5 & 0,040 & 2,500 & 0,183 & 69574,8 & 12702,7 & 316117,2 & 0,617 & 826072,7 & 11,87 \\
\hline 80 & + & 0,112 & 8,967 & 1,000 & 56872,1 & 56872,1 & 509955,5 & & 509955,5 & 8,97 \\
\hline \multicolumn{11}{|c|}{ Trienio 2009-2011 } \\
\hline 0 & 1 & 0,011 & 0,082 & 0,011 & 100000 & 1066,8 & 99020,7 & 0,989 & 7894274,3 & 78,94 \\
\hline 1 & 4 & 0,000 & 1,507 & 0,002 & 98933,2 & 194,3 & 395248,1 & 0,998 & 7795253,6 & 78,79 \\
\hline 5 & 5 & 0,000 & 2,500 & 0,001 & 98738,8 & 103,5 & 493435,4 & 0,999 & 7400005,5 & 74,95 \\
\hline 10 & 5 & 0,000 & 2,500 & 0,001 & 98635,3 & 118,6 & 492880,0 & 0,998 & 6906570,1 & 70,02 \\
\hline 15 & 5 & 0,000 & 2,500 & 0,002 & 98516,7 & 185,7 & 492119,2 & 0,998 & 6413690,1 & 65,10 \\
\hline 20 & 5 & 0,001 & 2,500 & 0,003 & 98331,0 & 253,4 & 491021,4 & 0,997 & 5921570,8 & 60,22 \\
\hline 25 & 5 & 0,001 & 2,500 & 0,003 & 98077,6 & 309,1 & 489615,1 & 0,996 & 5430549,4 & 55,37 \\
\hline 30 & 5 & 0,001 & 2,500 & 0,004 & 97768,5 & 401,7 & 487838,2 & 0,995 & 4940934,3 & 50,54 \\
\hline 35 & 5 & 0,001 & 2,500 & 0,006 & 97366,8 & 564,6 & 485422,3 & 0,993 & 4453096,1 & 45,74 \\
\hline 40 & 5 & 0,002 & 2,500 & 0,009 & 96802,1 & 830,5 & 481934,5 & 0,989 & 3967673,8 & 40,99 \\
\hline 45 & 5 & 0,003 & 2,500 & 0,013 & 95971,6 & 1258,4 & 476712,3 & 0,983 & 3485739,3 & 36,32 \\
\hline 50 & 5 & 0,004 & 2,500 & 0,020 & 94713,3 & 1889,9 & 468841,8 & 0,975 & 3009027,0 & 31,77 \\
\hline 55 & 5 & 0,006 & 2,500 & 0,030 & 92823,4 & 2816,2 & 457076,6 & 0,962 & 2540185,2 & 27,37 \\
\hline 60 & 5 & 0,009 & 2,500 & 0,046 & 90007,2 & 4097,5 & 439792,3 & 0,943 & 2083108,6 & 23,14 \\
\hline 65 & 5 & 0,014 & 2,500 & 0,069 & 85909,7 & 5951,5 & 414670,0 & 0,912 & 1643316,3 & 19,13 \\
\hline 70 & 5 & 0,023 & 2,500 & 0,108 & 79958,2 & 8639,7 & 378191,9 & 0,861 & 1228646,3 & 15,37 \\
\hline 75 & 5 & 0,038 & 2,500 & 0,174 & 71318,5 & 12402,8 & 325585,7 & 0,617 & 850454,4 & 11,92 \\
\hline 80 & + & 0,112 & 8,909 & 1,000 & 58915,7 & 58915,7 & 524868,7 & & 524868,7 & 8,91 \\
\hline
\end{tabular}

Fuente: Ministerio de Salud de Argentina, Dirección Nacional de Estadísticas e Información en Salud. Estadísticas Vitales. Buenos Aires: INDEC. 
